\title{
Patterning Abilities of First Grade Children: Effects of Dimension and Type
}

\author{
K. Marinka Gadzichowski \\ George Mason, Fairfax, USA \\ Email: Kgadicho@gmu.edu
}

Received July 18 ${ }^{\text {th }}$, 2012; revised August 20 ${ }^{\text {th }}$, 2012; accepted August 29 ${ }^{\text {th }}, 2012$

\begin{abstract}
In the United States children receive instruction on recognizing patterns beginning most often in kindergarten and continuing on through early elementary school years. Although widely accepted and included in curricula, patterning instruction has not been based on empirical research. The current study is the first attempt to determine how the dimension, e.g. color or shape, in which a pattern is displayed impacts children's ability to understand the pattern. This study is also an initial exploration of whether the overall "rule" of the pattern impacted a child's ability to recognize a pattern. Five types of patterns displayed in five different dimensions were presented to 204 first grade children in a completely counterbanced order. Results indicated that the dimension in which a pattern was displayed made no difference to the children. Patterns with alternating elements were significantly easier than any others, and those with increasing numbers of elements were significantly more difficult. Implications for instruction in patterning were discussed.
\end{abstract}

Keywords: Patterning; Cognitive Development; Education

\section{Introduction}

Children of elementary school age in most school districts in the United States are taught how to recognize patterns made up of letters, numbers, shapes and colors. This instruction is termed "patterning". There are several manuals in existence that provide directions on how to go about teaching patterning (Burton, 1982; Ducolon, 2000; Jarboe \& Sadler, 2003). The use of patterning in current curricula nationwide is based on a consensus of educators who believe that patterning has educational value because it improves children's cognitive abilities (e.g., National Council of Teachers of Mathematics, 1993). The improvement in thinking ability would lead to improved understanding of classroom instruction.

Suggested improvements in thinking include increased sensitivity to critical differences or sameness in items (Papic, 2007), identifying repetitions (alternations) and increases or decreases in the number of items (Economopolous, 1998), and detecting and applying relationships between items (Threlfall, 1999). These supporting abilities are thought to facilitate the development of prealgebra (Papic, 2007; Warren, Cooper, \& Lamb; 2006). There is no direct proof that this supposition is correct, but White, Alexander, and Daugherty (1998) found that mastery of patterns wherein elements alternated (e.g. red, blue, red, blue) was correlated with analogical reasoning, which would contribute in turn to an understanding of prealgebra.

The literature in this area is quite limited; there are only two studies that address how patterning instruction impacts achievement. In 1973, Herman gave 24 lessons on patterns made from alternating shapes, sizes or colors to kindergarten children from impoverished backgrounds. Her findings were that AfricanAmerican children who received this instruction made gains in numeracy skills but Latino/Hispanic children did not. In 2006, Hendricks, Trueblood, and Pasnak expanded the depth of pat- terning instruction to include 480 color, size, number, letter, and time (clock face) patterns ranging from alternations to unidimensional orderings to matrices presenting patterns in two dimensions. The children who received this type of instruction made greater academic gains than children in their control groups who received instruction focused specifically on academic material that had been developed upon the recommendation of teachers. These two studies constitute the entire set of empirical evidence that patterning has an effect beyond becoming better at patterning per se. However, it is clear that patterning instruction has become a fixture in education, and for that reason deserves investigation.

To date, very little research exists that has been designed to determine which types of patterns are the easiest or hardest for children to learn. It has been theorized that patterning was a stepping stone toward a very early form of analogical reasoning, and this type of reasoning was related to mathematical learning. (White, Alexander, \& Daugherty, 1998; Clements \& Sarama, 2007a, 2007b, 2007c). Patterning may also contribute to prealgebra since it is an early, age-appropriate form of instruction in rules and relations (Threlfall, 2004). Clements and Sarama (2007c: p. 507) theorized that "algebra begins with a search for patterns. Identifying patterns helps bring order, cohesion, and predictability to seemingly unorganized situations and allows one to recognize relationships and make generalizations." Therefore when children learn a patterning rule and then learn to apply that rule when they are presented with a pattern made up from new a child is showing "algebraic insight" - the understanding that a relation is not tied to particular concrete items. Hence, "recognition and analysis of patterns are important components of the young child's intellectual development because they provide a foundation for the development of algebraic thinking” (Clements \& Sarama, 2007b: p. 524). 
However, children transition from one type of thinking in preschool to a more complex cognition in early elementary school, and patterning incorporates many of the improvements in reasoning made during this time. Patterning may be intermediate between seriation and transitivity, In seriation, a child must understand how an item relates to the items that come before or after it in the sequence. When looking at patterns, this same rule applies. In seriation, the relation is a simple one. Either the item in question is smaller or larger than the other items in some dimension (height, width, weight, etc.). In patterning the relation is more complex; the relation might involve size, color, size and color, shape, or other dimensions that are more abstract, and the relationships can be multidimensional and much more complicated.

Transitivity is a more advanced reasoning ability. Transitivity is the understanding that if item $\mathrm{A}$ is related to item $\mathrm{B}$ in some way, and item $B$ is related to item $C$ in some way, then the relationship between $\mathrm{A}$ and $\mathrm{C}$ can be determined by comparing these items to item $\mathrm{B}$. The relationship between $\mathrm{A}$ and $\mathrm{C}$ (the key items) is not directly observed but rather deduced by comparing the key items to another item. Both transitivity and patterning incorporate the idea that an item is defined by, and simultaneously defines, properties of items that follow or preceed it. The primary difference between transitivity and patterning is that patterning does not require an individual to utilize the relations of $\mathrm{A}$ to $\mathrm{B}$ and $\mathrm{C}$ to $\mathrm{B}$ in order to determine the relation of $\mathrm{A}$ to C. Transivity does require that one use those relations. Since a child could make use of the transitive relation or alternatively make use of the simultaneous presentation of all the items and compare A to C directly, perhaps patterning is a precursor to transitivity.

When a child understands patterns of items in which items follow and precede other items, based on the rule of that pattern, then the child can make inferences about a neighboring item by looking at any one item in the pattern. A more advanced extrapolation would be to use a single item to make inferences about both of the neighboring items successively or simultaneously. Being able to compare the two inferences about the neighboring items in order to relate the neighboring items to one another would be considered transitivity.

A first step in understanding patterning as an aspect of cognitive development is to determine what kinds of patterns are easy for children and what kinds are difficult. Does the dimension-colors, shapes, letters, etc.-make a difference, or do children abstract the pattern rule independently of the dimension in which it is presented? Gadzichowski, Kidd, and Pasnak (2010) found that presenting preschoolers with oddity problems in different dimensions created large differences in the accuracy with which these children applied the same simple rule (the oddity principle). The same may well hold true for patterns early elementary school children confronted with the more complex rules involved in patterning. Further, not all of the rules which define patterns are equally complex. Which pattern rules do children grasp readily, without instruction, and which require improvement in the kind of inferences the children can make? Answers to these questions, which have never been asked by psychologists or educators, can inform investigations of how patterning relates to children's cognitive development, and also aid educators in determining what types of patterns can form the basis for the most fruitful classroom instruction. The present study is an effort to answer these questions by testing the following hypotheses:

Is children's recognition of the same pattern equivalent reg- ardless of the dimension which it is presented?

Are all pattern rules being tested in this study equally difficult for young children?

\section{Method}

\section{Participants}

Parental consent was obtained for 204 first-grade children from an urban school district. There were 91 female and 113 male participants. Of those participants 84 were African American, 73 were Hispanic/Latino, 32 were middle eastern, 5 were Caucasian and 10 were of an ethnicity other than those listed.

\section{Materials}

Because there are an infinite number of possible patterns, a subset to compare had to be selected. Five different types of patterns, subjectively estimated to range from easy to difficult were constructed using Power Point so they could be presented to the participants via a Dell Inspiron 1545 laptop. Each type of pattern was constructed using letters, numbers, colors, shapes and pictorial representations of objects (cars, flamingos, bees, etc.). The first type of pattern (Type 1) was termed, simple alternating "and used a rule of ABBABB, e.g., red, blue, blue, red, blue, blue. This is the type of pattern children are usually taught (Economopolous, 2008; Papic, 2007). A second type of pattern (Type 2) was potentially a more difficult alternation. It followed a rule of a constant alternating with a variable element AEAZAF, e.g., green, purple, green, blue, green, red and was termed "advanced alternation". The third type of pattern (Type 3) was termed "symmetrical" and followed a rule of AKGGKA, e.g. grey, black, orange, orange, black, grey. Another type of pattern (Type 4) followed a rule termed "increasing". The rule was $\mathrm{ABABBABBB}$, e.g., red, orange, red, orange, orange, red, orange, orange, orange. Finally, the last type of pattern (Type 5) was termed "arbitrary" and this included patterns such as: AJDXNA, or yellow, blue, red, green, gray, yellow.

\section{Procedure}

The patterns were presented one at a time to each child individually, and each child had unlimited time to respond. Each child was shown 25 patterns in all; five shape, five color, five letter and five objects patterns, one of each type for each of the five pattern rules. The order of the presentation was completely counterbalanced. The last item in each pattern was missing and the children were asked to select the correct answer from four possible options that were presented.

\section{Analysis}

Initial analyses were conducted with two factor ANOVA for correlated measures. Inasmuch as each participant's score on each pattern was a one or zero (right or wrong), the interaction of dimension and pattern type is the appropriate error term.

\section{Results}

There were no significant differences between the five different dimensions (letters, shapes, colors, numbers and objects), $\mathrm{F}(4,16)=1.23, p>.05$. There were however, significant differences between the different types of patterns, e.g., arbitrary, symmetrical, etc., $\mathrm{F}(4,16)=10.20, p<.001$. Subsequent LSD 
post hoc analyses revealed that Type 1 patterns (simple alternations) were significantly easier than Type 2 patterns, $p<.05$ and also significantly easier than Type 3 and Type 4 problems, $p<.005$ and $p<.001$ respectively. Type 4 (increasing) patterns were significantly more difficult than all other types of patterns, $p<.01$. There was no significant difference between pattern Types 2, 3 and 5 . The percentage correct for each dimension and type of pattern is presented in Table $\mathbf{1}$.

\section{Discussion}

Because the patterning instruction observed in local elementary schools was conducted almost exclusively on patterns constructed from shapes, colors and numbers, it was assumed that those dimensions would be significantly easier for the children. However, the first grade children were able to recognize patterns constructed using letters, numbers, colors, shapes or objects with equal accuracy. They did not have the difficulty applying the same rule to different dimensions that Gadzichowski et al. (2010) reported for preschoolers who attempted to apply the oddity principle to stimuli varying in color, size, shape, or orientation. It appears that by first grade, children are able to abstract relations between stimuli with less attention to their perceptual characteristics.

The most basic alternating patterns, e.g., red, blue, red, blue, red, blue or red, blue, green, red, blue, green are often taught toward the end of the kindergarten school year and the beginning of the first grade school year. Therefore, the findings that the participant's performance on such patterns (ABBABB) was better than performance on other pattern types makes sense.

The Type 4 patterns were the most difficult, indicating that the idea of a constant object alternating with an increasing numbers of objects is a complex concept and beyond the ability of most first grade children at the beginning of that academic year. Since the most common mistake in "solving" this type of pattern was to pick the answer choice that would have made the pattern a simple alternation, it appears that the idea of an increasing element was lost on the participants.

Perhaps the most surprising finding was that the Type 5 pattern problems were solved with almost as much accuracy as the ABBABB patterns. These Type 5 patterns had no discernible rule; a child would have to reach the understanding that the pattern is random and merely repeats itself.

In sum, these findings show that children's ability to understand patterns is not so much impacted by the dimension in which the pattern is presented, as it is by the over arching structure of the pattern itself. The implications for educators are two-fold. First, it appears that there need not be much concern about the dimension in which a pattern is presented. Second, alternation patterns are those with which instruction in patterning should start, because those are the easiest and would hence be best for

Table 1.

Percentage correct for five types of patterns presented in five different dimensions.

\begin{tabular}{ccccc}
\hline \multicolumn{5}{c}{ Dimension } \\
\hline Color: & Shape: & Object: & Letters: & Numbers: \\
61.27 & 51.67 & 62.75 & 57.45 & 54.90 \\
Type 1 & Type 2 & Type 3 & Type 4 & Type 5 \\
& & & & \\
ABBABB: & AEAZAF: & AKGGKA: & ABABBABBB: & AJDXNA: \\
86.18 & 64.41 & 55.69 & 19.80 & 67.84 \\
\hline
\end{tabular}

beginners. Third, and perhaps most important, patterning instruction should not stop with alternation patterns. The first graders in the present study had received patterning instruction in kindergarten, as part of the curriculum of the local school system, and perhaps in preschool as well. Yet they did not generalize to more advanced patterns, particularly those with increasing numbers of elements. Comprehending systematic increases is integral to mathematics, so it appears that instruction on these types of patterns, and on advanced types of patterns in general would be beneficial to children as they develop their mathematic skills.

Since pattern analysis is a cognitive skill that develops naturally, but has scarcely been investigated, and because it is also taught in American educational systems, developmental psychologists should not continue to neglect it. In order to better understand how it develops and how formal instruction in abstract cognition such as patterning impacts not only the development of that ability, but also other areas of cognition, patterning is a topic that should continue to be explored. Future research should explore other types of patterns, and attempt to further determine which patterns are easiest and which are harder so as to establish a more complete knowledge base for including patterning instruction in formal education.

\section{Acknowledgements}

The author acknowledges the constructive assistance of Robert Pasnak and the gracious participation and cooperation of Dr. Monte Dawson, Kristen Clark, and the principals, teachers, and students of the Alexandria City Public Schools.

\section{REFERENCES}

Lester Jr., F. K. (2007) Mathematics learning. Second handbook on mathematics teaching and learning (pp. 461-555). Charlotte, NC: Information Age.

Clements, D. H., \& Sarama, J. (2007c). Mathematics. In R. S. New, \& M. Cochran (Eds.), Early childhood education: An international encyclopedia (Vol. 2, pp. 502-509). Westport, CN: Praeger.

Ducolon, C. K. (2000). Quality literature as a springboard to problem solving. Teaching Children Mathematics, 6, 442-446.

Economopolous, K. (1998). What comes next? The mathematics of patterning in kindergarten. Teaching Children Mathematics, 5, 230-233.

Gadzichowski, K. M., Kidd, J. K., \& Pasnak, R. (2010). How odd is that? Poster presented at the meeting of American Psychological Science, Boston, MA.

Hendricks, C., Trueblood, L., \& Pasnak, R. (2006). Effects of teaching patterning to first graders. Journal of Research in Childhood Education, 21, 77-87. doi:10.1080/02568540609594580

Herman, M. L. (1973) Patterning before mathematics in kindergarten, doctoral dissertation, Columbia University, 1972. Dissertation Abstracts International, 33, 4060.

Jarboe, T., \& Sadler, S. (2003) It's as easy as 123: Patterns and activities for a creative, balanced math program. Peterborough, NJ: Crystal Springs BooksNational Councils of Teachers of Mathematics. (1993). Curriculum and evaluation standards for school mathematics Addenda Series, Grades K-6. Reston, VA: NCTM.

Papic, M. (2007). Promoting repeating patterns with young childrenMore than just alternating colors. Australian Primary Mathematics Classroom, 12, 8-13.

Threlfall, J. (1999). Repeating patterns in the early primary years. In A. Orton (Ed.), Patterns in the teaching and learning of mathematics (pp. 18-30). London: Cassell.

Warren, E. A., Cooper, T. J., \& Lamb, J. T. (2006). Investigating functional thinking is the elementary classroom: Foundations of early algebraic reasoning. Journal of Mathematics Behavior, 25, 208-223. 


\section{K. M. GADZICHOWSKI}

doi:10.1016/j.jmathb.2006.09.006

White, S. C., Alexander, P. A., \& Daugherty, M. (1998). The relationship between young children's analogical reasoning and mathemati- cal learning. Mathematical Cognition, 4, 103-123. doi:10.1080/135467998387352 